\title{
Chapitre 6 La catégorisation métalangagière en RDA
}

(1) $\mathrm{Il}_{\mathrm{i}}$ éructe : «Sans moi $\mathrm{i}_{\mathrm{i}}$, en tout cas!».

(2) Après moult salutations et protestations d'amitié, ils ont échangé des banalités sur le train du monde.

(3) Comment a-t-i $\mathrm{i}_{\mathrm{i}}$ pu s'aveugler ainsi, se lamente-t-i $\mathrm{i}_{\mathrm{i}}$.

(4) Si l'on en croit ses péremptoires affirmations, tout est réglé.

(5) Il est « retenu contre sa volonté » selon le délicieux euphémisme de nos amis anglais.

Dans ces énoncés de RDA les segments soulignés relèvent, dans leur variété morphologique et syntaxique, d'une même opération métalangagière - celle de la catégorisation qui, on le voit, ne se ramène pas, tant s'en faut, à la question des « verbes introducteurs».

Régulièrement évoquée comme dimension pertinente dans les présentations $\mathrm{du}$ « discours rapporté », objet, à juste titre, d'exercices pédagogiques, celle-ci relève de la catégorisation métalangagière, et les nombreuses études ${ }^{1}$ qui lui ont été consacrées présentent un ensemble consistant d'analyses et d'observations - tant au plan lexico-syntaxique (distribution dans les constructions $V$ parole : «... »/V parole que...) que lexico-sémantique (structuration en termes de traits sémantiques du champ lexical constitué) - sur ce type de catégorisation en RDA.

Mais il convient au-delà d'une focalisation sur les "verbes introducteurs » - qui n’apparaît pas sans rapport avec l'insistante tradition de la conception dérivationnelle $\mathrm{DD} \longrightarrow>$ DI et des exercices de transposition qui l'accompagnent - d'envisager la question de la catégorisation métalangagière en RDA dans toute son ampleur : on tentera de l'appréhender

- comme partie prenante du fait langagier général de la catégorisation métalangagière avec ce qu'elle tient de cette appartenance et ce qui l'y spécifie (1) ;

- puis, au-delà de ses formes par rection verbale en DD et DI, dans la diversité des configurations formelles (2) et la finesse du maillage lexical (3) à travers

\footnotetext{
1 Notamment, les structurations en termes de traits pertinents proposées par Wunderlich (1969), Strauch (1972), Charolles (1976), Kerbrat-Orecchioni (1980 : 100 sq.), le recensement d'un millier de verbes et locutions verbales proposé par Z. Nikodinovski (1986) - parfois peu usités - et, récemment, la constitution d'une base de données d'environ 700 verbes « introducteurs de citation », organisée selon des critères sémantiques à partir de la base Frantext et du journal Le Monde, $c f$. Mourad (2001), Mourad \& Desclés (2004).
} 
lesquels elle se réalise, comme dans son extension à tous les modes de la RDA, selon des pondérations inégales (4);

- et enfin comme variable pertinente au plan des discours, selon le mode sur lequel elle y est mise en œuvre (5).

\section{La RDA : secteur particulier de la catégorisation métalangagière}

« La langue peut, en principe, tout catégoriser et interpréter, y compris ellemême » : l'exercice de ce pouvoir de catégorisation réflexive, souligné par Benveniste ( $c f$. chap. 1.1), passe par l'existence d'un lexique métalinguistique permettant de nommer les faits de langage : sa richesse - balisée par J. Rey-Debove ${ }^{2}-$ dit combien nous importe l'univers de langage dans lequel nous vivons.

Remarque 1 : Métalexique et lexique « mondain ». Le lexique est réparti par cet auteur en trois ensembles : (I) mots mondains « destinés à parler de ce qui n'est pas le langage » (maison, chaud, respirer, mortellement...) ; (II) mots métalinguistiques « destinés à parler du langage » (adjectif, déclinaison, illisible, dire...), parmi lesquels des mots « à haute fréquence » (mot, dire, parler, écrire...) ; (III) mots neutres qui s'intègrent indifféremment aux deux ensembles précédents (il, que, forme, quand...). Sont signalés :

- $\quad$ les cas de double appartenance I/II par polysémie (conjuguer ses efforts/un verbe),

- le caractère non discret de l'opposition ( \pm trait langage), base de la répartition I/II, la notion de « densité métalinguistique » étant proposée pour rendre compte d'un continuum sur lequel se situeraient par exemple, dans un ordre de densité décroissante, les éléments : parole - parler-parleur - parolier - parloir,

- la question de l'inclusion (ou non) des mots renvoyant à une « opération de pensée » (idée, opinion, jugement) - évoquée ci-dessus chap. 2.

La catégorisation métalangagière - comme toute catégorisation - repose sur une prédication sous-jacente de l'ordre de : ce fait (langagier) est un / constitue un / relève de la classe des... Cette opération est à l'œuvre dans les énoncés suivants :

(6) « Acheter » et « vendre » sont des verbes converses ; «Vient-il ? » relève de l'interrogation totale; La classe des adverbes ( « bien, très, soudain,... ») est invariable.

(7) Ma réponse est « oui »; Je viendrai, ce n'est pas une promesse en l'air ; Je te conseille de ne pas y aller ; Pour conclure sur ce chapitre... ; ... si je puis risquer cette formulation métaphorique.

(8) Ce ne sont de sa part que reproches incessants, récriminations et jérémiades ; Le maire a confirmé que le projet était maintenu.

2 Rey-Debove (1978) chap. 3 Le Métalexique. 
mais le mode sur lequel elle s'effectue varie avec la nature du référent et le rapport de l’instance énonciatrice, « catégorisante », à ce référent.

Là où, en (6), le principe de la catégorisation du référent que sont les faits de langue («types », étrangers à l'univers des événements d'énonciation et du sens), stables, inventoriables, est celui de leur assignation univoque à des classes définies par un discours métalinguistique - quel que soit le degré de précision et d'explicitation de ces définitions, du discours spontané aux élaborations les plus contrôlées -, les référents de (7) et (8), « tokens ", événements singuliers porteurs de sens, ininventoriables, font, eux, entrer dans un régime de catégorisation interprétative. Mais sur cette base, commune, ARD (auto-représentation du dire) et RDA se distinguent foncièrement : en (7) la catégorisation s'articule à son référent dans le cadre d'un acte d'énonciation unique, " accompagnant » l'énoncé dans le moment de son énonciation ; elle en fait partie, elle participe à la production du sens de l'énoncé qui, en s'auto-catégorisant comme « réponse », " conseil », « conclusion » ou « formulation métaphorique »... s'institue comme tel ${ }^{3}$; c'est, au contraire, le décalage entre l'instance catégorisante et son référent - discours autre - qui caractérise, en (8), la RDA avec une double conséquence : d'une part l'interprétation inhérente à la catégorisation des " tokens » s'ouvre, sans limite, à tous les effets de l'altérité, autorisant, pour un $\mathbf{e}^{\mathbf{0}}$, des catégorisations variées en $\mathrm{E}$ (voire divergentes telles, ci-dessus, insulter vs critiquer dans l'échange de l'exemple (3) de l'introduction de cette partie III) ; d'autre part il faut, même si c'est une lapalissade, rappeler que là où la catégorisation (et plus généralement la représentation), c'est-à-dire l'image donnée d'un référent token est, dans l'auto-représentation, articulée au réel de ce token, par stricte co-présence ${ }^{4}$, le référent $\boldsymbol{e}^{\mathbf{0}}$ de la RDA n'y est présent, sans contrepoids aucun du côté du réel, que par l'image qui en est donnée. On peut par ailleurs observer que celle-ci met à contribution un lexique de catégorisation métalangagière plus riche que celui qui figure

3 Les verbes performatifs sont évidemment au cœur de cette configuration de catégorisation sui-référentielle, mais on voit qu'elle s'étend largement au-delà : du performatif canonique je te conseille de venir, à ses versions « dégradées » par détachement - Viens, c'est un conseil que je te donne ; Viens ! Ecoute mon conseil ; je vais te donner un conseil : viens... - ou encore je vous adresse une lettre de protestation/de recommandation, pour atteindre à des auto-catégorisations comme formulation métaphorique (7) ou ... et c'est un euphémisme; pour poursuivre mon argumentation/ma démonstration... qui ne présentent pas de « performativité ». ( $C f$. pour une réflexion sur le rapport entre performatif et quasi-commentaires - du type Viens, c'est un ordre - (Recanati 1981 : 48 sq.). 4 L'interlocution occupe une position d'entre-deux entre "le même » de l'ARD et l'autre de la RDA dans laquelle peut s'inscrire une successivité immédiate du référent et de sa catégorisation par un autre, comme dans le couple (1- 2a, introduction partie III) ou l'échange : « $\mathbf{L}_{1}$ : - Faites attention !. / $\mathbf{L}_{2}:-$ Vos menaces m'indiffèrent / Vos conseils me touchent ». 
dans les auto-catégorisations, s'étendant à des catégorisations que ces dernières ignorent : notons, par exemple - sans entrer dans l'étude comparative, qui serait utile - que les catégorisations plutôt dépréciatives, qu'elles portent sur la réalisation matérielle du dire - claironner, hurler, bafouiller, bégayer, etc. - ou sur son contenu - insinuer, colporter, raisonnement oiseux, avis mal intentionné, etc. - sont aussi usuelles en RDA qu'elles le sont peu pour le Discours en train de se faire.

\section{Configurations formelles de la catégorisation}

La catégorisation métalangagière concerne tous les modes de la RDA : il convient de le souligner - en deçà des différences observables entre les modes, et entre les formes d'un même mode quant à la pondération par rapport aux autres opérations métalangagières, de reformulation. C'est ce que montrent, $c f$. aussi (1) à (5), les exemples suivants (construits exclusivement avec des verbes de parole) :

(9) DD Il a supplié / ordonné : «Venez avec moi ».

(10)DI a Il a refusé / conseillé que Jean parte.

b Il a encensé / démoli le film de X.

(11)Bivocal $\quad \mathrm{Il}_{\mathrm{i}}$ était prostré. «Comment avait-il $\mathrm{l}_{\mathrm{i}}$ pu être aussi naïf ? » se lamentait-il $\mathrm{l}_{\mathrm{i}} /$ rageait-i $\mathrm{l}_{\mathrm{i}}$. (12)MAS Le réchauffement climatique est, d'après ce que prophétisent les écologistes / démontrent les études récentes, à prendre au sérieux.

(13)MAE Il fait partie de ce que l'on a baptisé / nommé ironiquement la « gauche caviar ».

Elle ne se cantonne pas à la forme du verbe de parole recteur, selon le schéma canonique : il a dit : "Il fait beau »/qu'il faisait beau. Elle puise largement au lexique métalinguistique des noms, et passe par toutes les configurations du « cadrage » énonciatif de $\boldsymbol{e}$ : incises, appositions, anaphores, cataphores, lien sémantique.

\section{Verbes de parole}

En ce qui concerne les verbes de parole, ils entrent, outre la construction en verbe transitif recteur, dans deux autres configurations majeures : l'une (a), syntaxique, de construction d'un e avec une incise, l'autre (b), sémantique, d'articulation interprétative d'un $\boldsymbol{e}$ avec un verbe n'ayant pas le statut syntaxique d'un introducteur, recteur d'un « $\boldsymbol{e}$ » complément d'objet, mais le rôle « d'annonceur » d'un « $»^{5}$. La construction avec introducteur :

5 On retrouve ci-dessous chap. 8.2.1.3, p. 256 sq. la distinction - sans aucun doute à affiner - entre introduit et annoncé dont l'incidence ne se limite pas à l'étendue lexicale de la catégorisation. 
(a) Jean a promis « $\mathrm{e} »$

et la configuration avec annonceur :

(b) Jean a tempêté ; « e »,

s'opposent quant à la possibilité pour le $\boldsymbol{e}$ d'être pronominalisé en le, ou d'occuper la place de sujet au passif :

(a') «e », Jean l'a promis, «e » a été promis par Jean

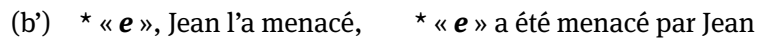

Dans les exemples $(29,31,37)$ chap. 5, par exemple, les verbes appelait, reprenait, sourit, fonctionnent comme « annonceurs » du « $e$ » qu'ils précèdent

Si on les évoque ici - sans entrer dans l'analyse de leur fonctionnement - c'est en tant que les ressources lexicales offertes à la catégorisation y sont largement étendues (15), les verbes (incluant les verbes de pensée) n'y étant pas soumis aux restrictions propres à la rection (14) :

(14) * Il sermonne qu'on n'a pas fait comme il avait dit ; *Il s'effarouche qu'il n'osera jamais le faire.

VS

(15) a Il sermonne : Vous n'avez pas fait comme j'avais dit ; Il s'effarouche : Je n'oserai jamais le faire!

b Vous n'avez pas fait comme j'avais dit, sermonne-t-il ; Je n'oserai jamais le faire ! s'effarouche-t-il.

Et ces configurations se réalisent en dehors du DD, notamment au mode bivocal :

(16) « On n'a pas fait comme $\mathrm{il}_{\mathrm{i}}$ avait dit », sermonne-t-il $l_{\mathrm{i}}$ / $/ \mathrm{Il}_{\mathrm{i}} \mathrm{s}^{\prime}$ effarouche : « Jamais $\mathrm{il}_{\mathrm{i}}$ n'osera le faire!».

Au-delà des verbes de parole ${ }^{6}$ ou de sentiment, l'élargissement de la catégorisation dans l'incise à des Verbes exclus comme verbes recteurs touche à ce qui

6 Sans entendre par là que cet ensemble soit univoquement déterminable ; ainsi Fiala (1992) relève-t-il pour aborder des emplois comme « verbe de parole » dans Le Neveu de Rameau : «Un après dîner, j'étais là, [...] lorsque je fus abordé par un des plus bizarres personnages de ce pays [...]. [...] Il m’aborde... Ah ! ah ! vous voilà Monsieur le philosophe [...]. » ; rappelant la définition de l'Encyclopédie : « On aborde les personnes à qui l'on veut parler» (idt), il le traite, dans ce cas, comme un verbe de mouvement porteur d'un trait de dire. 
relève (gestes «parlants ») du mimo-gestuel tendanciellement codifié accompagnant le dire ${ }^{7}$ :

(17) ... hausse-t-il les épaules ; hoche-t-il la tête ; sourit-il ; lève-t-il les sourcils d'un air interrogatif ; fait-il la moue ; ...

\section{Noms de parole}

Le lexique métalinguistique où puise la catégorisation métalangagière en RDA ne se restreint pas au verbe, on l'a dit, mais relève régulièrement du nom (morphologiquement couplé à un verbe de parole : répondre/réponse ; blâmer/blâme... ou non : hymne, lapsus, proverbe, topo, tirade, etc.). Comme pour le verbe, l'articulation d'un nom au $\boldsymbol{e}$ dont il assure la catégorisation peut relever d'un rapport syntaxique ou d'un lien sémantique.

Relèvent du rapport syntaxique :

- la relation attributive du type Nméta est e ou e est Nméta, couramment observable en DD, DI et sans exclusion du Bivocal

(18) a Sa réponse a été : «Je ne viens pas » / qu'il ne venait pas.

b « Je ne viens pas» / Qu'il ne venait pas a été sa seule réponse.

c Allait-il $\mathrm{l}_{\mathrm{i}}$ arriver à temps ? était la question qui le $\mathrm{e}_{\mathrm{i}}$ taraudait.

- la relation appositive entre un $\boldsymbol{e}$ et un Nméta :

(19) L'annonce du ministre, « le projet est maintenu », a rassuré.

(20) La cuisine des femmes [...] rien n’y est « masqué » (expression de la cuisine des chefs). [La Reynière, Le Monde des loisirs, 04-05-1985]

- la rection nominale d'une complétive (DI) ou d'une relative en selon par un Nméta :

(21) La promesse que / selon laquelle le projet est maintenu, a rassuré.

7 Lorsque les incises s'étendent à tout geste, attitude, voire activité de $\boldsymbol{l}$ en rapport ou simultané à son dire, comme : « se leva-t-il brusquement ; s'assit-il lourdement ; pianote-t-il sur la table ; range-t-il son portefeuille... », il n'est plus question de les assimiler à des verbes de parole : on quitte les rives de la catégorisation métalangagière. C'est ce dont, à sa manière, rend compte $\mathrm{D}$. Wunderlich (1969) dans son analyse, dans un cadre de grammaire générative, du champ lexical des Verba Dicendi, en proposant de décrire les énoncés de DD avec incise ou « annonceur » comme résultant d'une transformation d'effacement (ou d'ellipse) à partir d'une « structure profonde » coordonnant un verbum agendi à un verbum dicendi ayant le même sujet personnel et le même temps : « $\mathrm{il}_{\mathrm{i}}$ lève le poing et $\mathrm{il}_{\mathrm{i}}$ dit : « ... » => il lève le poing : « ... », ou « ... », lève-t-il le poing ». 
relève d'un lien sémantique co-référentiel :

- la cataphore (associée (a) ou non (b) à un verbe de dire " neutre »), en DD et bivocal :

(22) a Le président a fait une / cette promesse : " Les impôts vont baisser ».

b Promesse du président : « Les impôts vont baisser ».

c Réponse de Marie $_{\mathrm{i}}$ : « Elle $_{\mathrm{i}}$ n’y a pas pensé ! »

- et, massivement, l'anaphore, qui entre dans deux configurations. Dans la première (23), observable avec tous les modes de RDA, elle apporte une spécification, un enrichissement à la catégorisation déjà présente dans la phrase (de RDA) qui précède :

(23) a DD Jean a dit : « Bien sûr que j'y arriverai. ». Cette rodomontade a fait rire.

b DI Le président a dit qu'il allait baisser les impôts. Cette promesse sera-t-elle tenue ?

c Bivocal « Ce n'était pas sa faute, disait-il, il n'avait pas été prévenu ». Cette pitoyable défense ne convainquit personne.

d MAS D'après ce qu'a dit Jean, les impôts vont baisser. C'est une information à prendre avec circonspection.

e MAE Là se met en place ce que les nazis appellent la " solution finale ». Cette ignoble périphrase euphémisante a été inventée en [...].

Dans la seconde (24) dont seul le DI semble exclu (cf. ci-dessous 4.), l'anaphore apporte une catégorisation rétro-active à la séquence $\boldsymbol{e}$, vierge de catégorisation, à laquelle elle succède :

(24) a DD «Venez avec moi ». Cet /l'ordre de X a paru menaçant / Cette / la supplication de X a ému. (comparer (9))

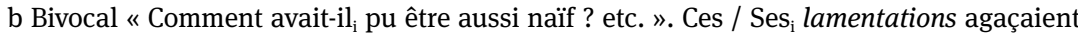
son associé. (comparer (11))

c MAS Le réchauffement climatique serait à prendre au sérieux. Les prophéties des écologistes semblent se vérifier. (comparer (12))

d MAE Mr X est « retenu contre sa volonté ». Cet euphémisme tout britannique ne doit pas masquer le fait de la prise d'otage. (comparer (5))

Mécanisme de progression discursive - qui mérite l'attention pédagogique au même titre que l'entraînement aux verbes introducteurs ${ }^{8}$ - cette anaphorisation métalangagière apparaît aussi présente dans le fonctionnement de la presse écrite (Moirand 1975) que dans le déroulement narratif, celui des Fables de La

$8 \mathrm{Cf}$. dans (Jespersen et al. 1997) la place que réserve la « séquence didactique " visant l'argumentation via le discours rapporté aux exercices "d'introduction de nominalisation » - cette déclaration, ce souhait, ses affirmations... - , et l'enrichissement de ces « anaphores résomptives » par des adjectifs évaluatifs. 
Fontaine, par exemple, dont M.-J. Béguelin (1998) tire un séduisant florilège de «SN démonstratifs métalinguistiques » renvoyant à des RDA antécédentes (principalement au DD, avec un DI, un Bivocal) :

cette harangue, cette oraison funèbre, cet avis-là, ce conseil, cette offre, cette adroite répartie, ce serment, cette pensée...

À côté de ces formes - syntactico-sémantiques, intra et interphrastiques - par lesquelles un élément catégorisant entre en relation avec une séquence $\boldsymbol{e}$, verbes et noms métalangagiers constituent aussi le noyau sémantique de $R D A$ purement catégorisantes, sans élément $\boldsymbol{e}$ isolable. Ainsi de ces trois types de RDA, susceptibles d'apparaître en $\mathrm{X}$ dans un contexte comme :

(25) On a demandé au maire quand les travaux seraient finis. ...X... On est passé à la suite.

avec $\mathrm{X}=$ (a) Il a répondu évasivement.

(b) Réponse évasive ; Sa réponse évasive n’a pas surpris.

(c) Il a fait une réponse évasive.

On peut souligner, ici encore, l'importance du lexique nominal susceptible d'entrer dans les combinaisons, très usuelles, verbe d'action neutres $+\mathrm{N}$ de catégorisation métalangagière ${ }^{9}$, telles

faire des plaisanteries, promesses, aveux, commentaires... ; donner des ordres, prodiguer des conseils... ; lancer des piques ; multiplier, accumuler les bons mots, paradoxes, prétextes, sous-entendus ; échanger des mono-syllabes, injures, politesses...

\section{Le maillage lexical de la catégorisation spontanée des dires}

La catégorisation métalangagière en RDA dispose d'une grille lexicale serrée : par rapport à la neutralité du verbe dire, et au il dit, remplissant, au degré minimal de la catégorisation métalangagière, la fonction purement « indicatrice » du fait d'un dire $^{10}$, la spécification du dire représenté dispose d'un ensemble considérable de lexèmes - verbes et noms, principalement, mais aussi adjectifs et adverbes - por-

9 « neutres " est pris ici au sens de Rey-Debove, $c f$. ci-dessus, Remarque 1. La construction $V+$ Nom méta est couplée à un $\mathrm{V}$ (ce qui ne veut pas d'ailleurs dire synonymes, $c f$. plaisanter / faire des plaisanteries) ou non : V+bons mots, monosyllabes, paradoxes...

10 " verbe introducteur fournissant l'information minimale, autrement dit [...] signal de reproduction à l'état pur » selon (Strauch, 1972 : 227), qui propose de l'appeler « verbe introducteur simple »; notons cependant (cf. 4. ci-dessous) que le DI constitue pour dire un contexte spécificateur excluant de renvoyer à une interrogation. 
teurs du trait sémantique « dire/discours », de façon essentielle (a) ou dans une acception régulière de leur polysémie (b), tels ${ }^{11}$ :

a) concis, péremptoire, verbeux, prolixe, injurieux, pédant, docte, pontifiant, captieux, diffamatoire, compendieusement, textuellement, texto, sic...

b) fallacieux, paradoxale (formulation), provocateur (discours), confus, fumeux, obscur, limpide, clair succinct (discours, propos)...

Loin de l'exhaustivité d'un inventaire, autant que de la systématicité d'une structuration du champ en termes de traits pertinents, le balisage qui suit, dégageant quelques uns des plans selon lesquels et à l'intérieur desquels s'opère la catégorisation lexicale des faits de dire, voudrait avant tout faire apercevoir la finesse $\mathrm{du}$ « maillage » à travers lequel passent les représentations spontanées du dire en RDA.

Proposés comme exemple de catégorisation située à l'un ou l'autre des plans distingués par la représentation spontanée du dire, les lexèmes $\mathrm{V}, \mathrm{N}$ relèvent souvent de plusieurs de ces plans : rétorquer, par exemple, relève à la fois (dans le classement ci-dessous) de la réaction au dire de l'allocutaire [3] et d'un mouvement argumentatif [7] ; calomnier de dire du mal [6] et du dire jugé faux par L [7] ; etc.

Ce sont les verbes - plutôt que les noms correspondants - qui, pour suivre l'usage, sont mentionnés ci-dessous ; mais faisant appel à la compétence lexicale du lecteur, ils sont à entendre - hormis les rares cas où le verbe de dire ne connaît pas de dérivation nominale, par suffixe ou déverbal : morigéner, exciper..., par exemple - comme renvoyant au couple verbe et nom, les noms désignant l'action (de dire) et/ou son produit (le « dit » réalisé). Les verbes cités en [1] ci-dessous, par exemple, valent aussi pour écriture, écrit, fax, griffonnage, calligraphie, tag... Des $\mathrm{N}$ sont cependant aussi mentionnés : lorsqu'il n'existe pas de verbe correspondant (refrain, serment, poème...), lorsque leur sens s'est sensiblement autonomisé par rapport au verbe, notamment comme dénomination d'un genre de discours institué (ordonnance, par rapport à ordonner, supplique par rapport à supplier...), ou simplement lorsqu'ils m'ont paru d'un emploi plus usuel que le verbe (testament par rapport à tester...).

11 Par opposition, sans que le partage soit discret, des lexèmes tels que : long, bref, désagréable, beau, surprenant... qui, pour être évidemment à même de modifier des $\mathrm{N}$ (ou V) métadiscursifs, ne seront pas considérés pour autant comme présentant une acception métadiscursive. 
[1] En deçà de la représentation de « ce que » dit l'énoncé, un premier plan où se situe la catégorisation est celui de la réalisation matérielle, scripturale vs vocale du dire produit ou reçu :

crier, vociférer, claironner, articuler, grommeler, chuchoter, chantonner, ânonner, bégayer, bredouiller, babiller... ; écouter, entendre... ; avec (sur, d')un(e) ton, accent, intonation, voix, inflexion ${ }^{12}$... ; indiquant pour l'oral un rapport à l'écrit (visée/source) dicter, épeler, réciter ; écrire, faxer, twitter, griffonner, calligraphier, taguer... ; lire, déchiffrer, parcourir... ; en capitales, italique, caractères énormes, à l'encre... ; affiche, écriteau, étiquette, inscription, sms...

La fréquence avec laquelle les noms de genre de discours comportent l'indication du canal par lequel ils se réalisent, atteste de la place faite dans le lexique à cet élément : tract, préface, faire-part, missive... vs conversation, prêche, allocution...

La représentation en RDA de ce plan de la réalisation physique du dire - scripto / phono-gestuel - relève, de façon tout à fait privilégiée, parmi les autres aspects du dire, de cette opération de catégorisation. Ainsi :

(26) a L'adjudant, sa liste à la main, aboyait les noms.

b Dans un murmure presque inaudible, il admet connaître l'accusé.

(27) Il tressaillit quand une voix douce lui dit tout près de l'oreille - Que voulez-vous ici mon enfant? [Stendhal, Le Rouge et le Noir]. « Ne touchez pas les petits, bégaya-t-elle, vous êtes si laid!» Elle accentua ce mot avec un si étrange mépris que [...] [Zola, La Faute de l'Abbé Mouret ${ }^{13}$.

L’autre possibilité, celle d'une représentation mimétique de l'énoncé, permise par l'opération d'autonymisation, apparaît dans le fonctionnement usuel des modes autonymisant de RDA comme un cas marqué - chargée de dérision, agressive ou complice - dans des imitations à l'oral, d'une voix, d'un accent ou d'un défaut de prononciation, et d'une ostentation de fidélité dans les fac-simile qui, dans la presse (Le Canard enchaîné, par exemple) font « preuve à l'appui » pour des révélations.

12 Ces éléments, qui ont le plus souvent le statut d'expansion de $\mathrm{V}$ ou $\mathrm{N}$ de dire, peuvent aussi constituer, seuls, des " introducteurs " de $\mathbf{e}$, au DD notamment : comparer à quelques lignes d'intervalle «[...] et il affirma d'un ton de libéral que [...] » et « M. Dambreuse aperçut Martinon et, s'approchant de sa femme, d'une voix basse :/- C'est vous qui l'avez invité ? » [Flaubert, L'Éducation sentimentale, II, 2]. On notera par ailleurs que parler ne présente pas le trait « vocal» dans tous ses emplois, $c f$ : : Dans ce traité, $X$ parle de...

13 Exemples empruntés à P. Dufour (2004: 46-70), dans le riche chapitre qu'il consacre à la représentation romanesque du « Dialecte Corporel ». Pour un traitement approfondi et aigu de la question des « représentations écrites de l'oralité » voir Mahrer (2014), (2017) et, spécifiquement sur la catégorisation de l'oral, Mahrer (2017 : 222-245). 
Les discours se différencient fortement par la place qu'ils font, en RDA, à cette couche matérielle du dire - ignorée des genres pour lesquels les dires sont envisagés comme véhicules de savoir, elle apparaît notamment avec ceux qui font place à la narrativité : ainsi peut-on mettre en regard les Souvenirs de la Cour d'Assises d’André Gide qui parcourent toute la gamme des voix :

trébuchante, déplaisante, si faible que, pathétique, douce, nette et sèche, sourde, ton de réprimande presque douce, etc. [p. 623 sq. éd. de La Pléiade]

avec les comptes rendus de décisions de justice figurant dans les répertoires de jurisprudence et qui - notamment ceux des arrêts de la cour de cassation statuant sur ce qu'a déclaré, condamné, rejeté, etc. un tribunal - qui, saturés de RDA, en ignorent évidemment la dimension corporelle.

C’est aussi, pour des textes attentifs à la dimension matérielle du dire, par la pondération entre les deux voies de la catégorisation (normalement dominante) et de l'imitation phonique ou graphique (via l'autonymisation) que se spécifient des styles singuliers de parole ou d'écriture, notamment romanesques ${ }^{14}$ : pensons à la collection hétéroclite de documents, reproduits matériellement, dont Perec accompagne le déroulement de sa «Vie Mode d'emploi », ou, posant la question du transcodage, le bredouillement sélectif délibéré du père Grandet, ou les accents germaniques de Nucingen et de Schmucke ${ }^{15}$, mis en scène par Balzac (en DD, MAE) jusqu'à la fatigue du lecteur.

[2] Un second élément de catégorisation est celui du pôle l ou $\boldsymbol{r}$ de la co-énonciation à partir duquel est représenté l'acte $\boldsymbol{a}$ : si, conformément au canonique « il a dit », c'est la production d'énoncé qui est privilégiée, les $\boldsymbol{a}$ saisis sous l'angle de la réception relèvent pleinement de la RDA. Ainsi lire, entendre, par exemple, fonctionnent-ils comme « verbes introducteurs » de DI ou DD :

J'ai lu en quelque endroit que... (forme chère à La Fontaine)

Entendu sur TF1 : «...».

et peuvent-ils intégrer l'ensemble dit des « verbes de parole », si l'on prend le terme parole dans l'extension maximale (indifférente tant à l'opposition émis-

14 Voir, par exemple, Dufour (2004 : 63) notant l'incidence, dans les écritures romanesques, des places respectives accordées à la « phrase sinueuse qui cerne la complexité des signes non-verbaux [entendre non morphématiques] » (catégorisation) et à la « reconstitution d'un équivalent verbal au style direct » (autonymie à dimension imitative).

15 Dans Splendeurs et misères des courtisanes, par exemple, et dans Le Cousin Pons. 
sion / réception qu'à celle de oral / écrit) qui est celle du « fait de parole », ou du couple langue / parole. Dans cet ensemble, si lire, déchiffrer, par exemple, renvoient univoquement à un message, il n'en va pas de même pour :

entendre, écouter, capter, prêter l'oreille, saisir (au vol), voir, parcourir (des yeux)...

et autres verbes de perception auditive ou visuelle dont le fonctionnement métalangagier dépend de la mise en jeu, interprétative ou explicite, dans le contexte d'un élément de parole :

J'ai écouté le président ; j’ai écouté le discours ; c'est agaçant d'entendre dire que... ; c'est une expression qu'on entend souvent ; j'aime entendre parler de...

[3] Encore extérieur au contenu même du dire (mais susceptible de se combiner à la représentation de ce dernier), un second plan spécifie la place de l'acte a représenté par rapport à un autre dire. S'y distinguent, lexicalisés en couples V/N ou en $\mathrm{N}$ autonome, les facettes :

- du dire élément d'un dire :

introduire, ajouter, poursuivre, reprendre, conclure ; exorde, avant-propos, prologue, incipit, post-scriptum, envoi, codicille... ;

- du redire :

répéter, réaffirmer, citer, recopier, radoter, seriner, ressasser... ; refrain, proverbe, slogan, cliché, stéréotype, rengaine, potin, ragot... ;

- du dire-réaction :

couper, interrompre, répondre, répartir, acquiescer, répliquer, rétorquer... ;

- du dire portant sur un dire :

commenter, résumer, interpréter, analyser, paraphraser, traduire... ;

- du dire-échange :

dialoguer, bavarder, négocier, débattre, discuter, se disputer, s'entretenir... ; algarade, clash...

Au-delà de ces trois plans, la majeure partie du lexique métadiscursif de la RDA s'attache à ce qui s'y dit, faisant apparaître, à travers actes illocutoires, mouvements argumentatifs, opérations rhétoriques ou discursives, des traits divers :

[4] la saillance du destinataire dans les actes - et les genres - de l'interrogation, l'injonction, l'apostrophe, etc...

interroger, questionner, demander si, s'enquérir... ; ordonner, enjoindre, proscrire, interdire, permettre, (dé)conseiller, exhorter, implorer, prier, exiger, demander de... ; apostropher, invoquer, invectiver, insulter... ; menacer, défier, persifler, flatter, admonester, réprimander, morigéner, sermonner, féliciter, pardonner, encourager, dissuader, convaincre, avertir, prévenir... ; 
interrogatoire, questionnaire, interview, devinette... ; sermon, remontrance, harangue, ordonnance, prière, compliment, boniment, supplique, recette, homélie, bulle, requête...

[5] l'engagement au sens juridico-moral de $\boldsymbol{l}$ dans son dire : jurer, promettre, engager, attester, témoigner, certifier... ; serment, certificat, testament, déposition ...

\section{[6] l'appréciation (en bien/mal) portée par $\mathbf{l}$ sur ce dont il parle:}

blâmer, reprocher, dénigrer, dénoncer, accuser, médire, condamner, fustiger, maudire, vitupérer, incriminer, (se) moquer (de), railler, louer, célébrer, encenser, prôner, justifier, excuser, plaider pour... ; diatribe, critique, sarcasme, réquisitoire, éloge, panégyrique, dithyrambe, plaidoirie...

et, relevant du lexique des sentiments susceptible d'être annexé interprétativement au champ du dire :

souhaiter, se réjouir, s'enthousiasmer, regretter, déplorer, s’indigner, redouter, craindre...

\section{[7] la spécification de l'assertion de l}

\section{- en :}

remarquer, noter, indiquer, signaler, observer, constater, souligner, annoncer, informer ; opinion, avis...

- en fonction du mouvement argumentatif effectué :

affirmer, confirmer, soutenir, alléguer, admettre, convenir, concéder, reconnaître, nier, contester, objecter, récuser, refuser, s'opposer, contredire... ; argutie, argument...

- en fonction du jugement de vérité porté sur elle par $\mathbf{L}$ :

Vrai

révéler, confesser, avouer, prouver, montrer... ;

\section{Faux}

mentir, prétendre, diffamer, calomnier, dérailler, divaguer, délirer, vaticiner... ; prétexte, contrevérité, sophisme, balivernes, galéjade, rodomontades, foutaises, bobards, racontars... ;

\section{Incertain}

supputer, postuler, hasarder, prédire... ; hypothèse, conjecture ...

\section{- par l'implicitation, mise au jour par $\mathbf{L}$ :}

insinuer, sous-entendre, laisser entendre, faire comprendre, faire allusion...

et relevant du lexique des « pensées » susceptible d'être annexé au champ du dire :

juger, croire, estimer penser, trouver, point de vue, opinion, avis... 
[8] le type rhétorico-discursif dont relève le dire autre :

expliquer, exposer, analyser, résumer, développer, exemplifier, illustrer, détailler démontrer, argumenter, disserter, énumérer, comparer, commenter, raconter, narrer, décrire ... ;

et tous les noms de genres de discours correspondant à un ou plusieurs de ces types :

récit, anecdote, roman, manuel, recette, notice, mode d'emploi, dictionnaire, argumentaire, traité, conférence, article, éditorial, titre, poème, sonnet...

[9] Enfin, en deçà de ces dimensions illocutoires, argumentatives, rhétoriques, ... un ensemble de catégorisations se situe (notamment dans les modes autonymisants de la RDA dont la représentation met en jeu les «manières de dire ») au plan des opérations de nomination ou de formulation accomplies par $\boldsymbol{l}$ :

ce quel/commel le nomme, appelle, baptise, désigne, qualifie, formule (avec des expansions spécifiquement métadiscursives ou non comme joliment, improprement, par euphémisme...) ; paradoxe, périphrase, circonlocutions, galimatias, antiphrase, litote, métaphore, lapsus... (assortis d'expansions telles que : ampoulé, choisi, vulgaire, archaïque...)

- en annexant à la catégorisation des dires des éléments relevant du métalexique de la langue, dès lors que le contexte les associe à une énonciation : phrase, mot, terme, expression, locution, verbe, nom, monosyllabe..., dans des constructions du type selon/ pour reprendre le ... de $\mathbf{l}^{16}$.

On voit, à travers ce survol, l'étendue des claviers sur lesquels joue l'opération de catégorisation métalangagière, composante à part entière de la représentation interprétative, par $\mathbf{L}$, du sens des énoncés qu'il représente. Au déploiement usuellement invoqué des représentations jouant des ressources de la reformulation - paraphrastiques et/ou autonymisantes - :

(29) Le pape a dit : « vive l'abstinence » / « l'abstinence élève l'âme » / que l'abstinence était l'idéal / qu'il fallait préférer l'abstinence / etc.

répond, opérant sur un autre mode, l'éventail, d'un autre type mais aussi large, de la catégorisation :

(30) Le pape a loué/ fait l'éloge de / célébré / chanté les mérites de / rendu hommage à / recommandé / prôné / plaidé pour... l'abstinence.

Et cet éventail est d'autant plus large que, certes dépendantes des ressources lexicales métalangagières, les possibilités de la catégorisation sont, par le jeu des

16 Les lexèmes cités ici sont les plus fréquemment rencontrés, mais si l'on a peu de chances de voir dans les catégorisations de la RDA des termes comme déclinaison, conjugaison, subordonnée..., on ne peut rien exclure a priori et on rencontre facilement préfixe, suffixe, conditionnel, diminutif, pluriel... 
constructions associant plusieurs lexèmes métalangagiers et leurs expansions, illimitées. Le cumul d'éléments catégorisants, notamment, au principe de stéréotypes de RDA tels que :

(31) proférer des contrevérités / insinuations diffamatoires / allégations mensongères / preuves irréfutables etc.

est à l'œuvre dans tous les types de discours, à travers des formes diverses de catégorisation complexe :

(32) M. Marion fustige - le mot est faible - en termes sévères l'absence de consigne à la DGSE [...] [Le Monde, 26-03-1991].

(33) Un loup quelque peu clerc prouva par sa harangue / Qu'il fallait [...] [La Fontaine, Fables, VII-1].

(34) Je hasardai un conseil de transport immédiat dans un hôpital [...] [Céline, Voyage [...], p. 260].

(35) - Bonjour, docteur, lui dit Rodolphe.

Le Médecin, flatté de ce titre inattendu, se répandit en obséquiosités, et l'autre en profita pour se remettre un peu [Flaubert, Madame Bovary, partie II, chap. IX].

À travers le classement ébauché ci-dessus, à fondement sémantique, apparaît la richesse extrême du champ lexical de la catégorisation des dires ; au delà est à dessiner le réseau, relevant centralement de la "variété », de différenciations portant la finesse de son maillage lexical : par exemple vocabulaire général vs lié à une technique : courrier vs tweet, sms ; attaché à une sphère d'activité : bulle (papale), main courante (policière), éditorial... ; relevant d'un état de langue ancien : édit, placet ; d'émergence récente : éléments de langage, petites phrases, etc.

Parmi les foisonnantes problématiques discursives qu'ouvre ce territoire de la catégorisation des dires, on notera la question des modalités selon lesquelles, dans un domaine discursif particulier, s'opère, à lexème constant, le passage, l'accès, la « promotion » d'un nom métalinguistique « ordinaire » à un autre statut, plus étroitement normé. Il en va ainsi des noms de genres de discours ${ }^{17}$ émergeant de l'ensemble plus vaste des "noms de discours » : sans envisager la complexité des enjeux de nomination des genres littéraires, pensons à ces "sur-catégorisations » normatives propres aux espaces institutionnels, instituant, dans la catégorisation métalangagière commune d'un dire comme résumé, commentaire ou question,

17 Problématique éclairée notamment dans Branca-Rosoff (dir., 1999), Petit et Beacco (2004) ou Mellet et Sitri (2010). Notons aussi l'intéressant dossier (Krieg-Planque et al., 2011) analysant le fonctionnement dans la sphère médiatico-politique du « travail de catégorisation » constituant tel propos d'un homme politique en « petite phrase », livrée comme telle - décontextualisée - à son ressassement médiatique. 
un nom de genre (scolaire) du « Résumé » ou du « Commentaire », ou (parlementaire) de «Question au gouvernement ». Il en va de même dans le fonctionnement judiciaire, où débats et sanctions peuvent avoir pour enjeu certaines de ces catégorisations métalangagières, promues - via définition et jurisprudence - au statut de catégorie juridique permettant la « qualification » d'un acte langagier comme « diffamation, insulte à agent dans l'exercice de ses fonctions, menace de mort, etc. », par exemple.

\section{La catégorisation dans les divers modes de RDA - son affinité avec le DI}

Tous les modes, on l'a dit, sont concernés par l'opération de catégorisation, mais ils ne le sont pas également : le DI, tel qu'il a été défini ${ }^{18}$, présente, vis à vis des quatre autres modes, un rapport différent à l'opération de catégorisation, à laquelle il apparaît « lié ».

Trois points sont, de ce point de vue, à signaler.

\section{Le DI requiert la catégorisation}

là où les autres modes présentent une panoplie de formes sans aucun élément catégorisant. Outre l'ensemble des variantes strictement non marquées de ces modes (l'allusion pour la MAE, le DDL pour le DD...), citons, par exemple, ces formes marquées - guillemets, mention d'une source - mais non-catégorisées :

(36) DD Jean : « Je ne viens pas »; Il arrive. «Vous allez bien ? » et il repart.

Bivocal $\mathrm{Il}_{\mathrm{i}}$ '’assied. «Qu'on vienne $\mathrm{le}_{\mathrm{i}}$ chercher, $\mathrm{il}_{\mathrm{i}}$ n'ira pas plus loin! »

MAS Il a, selon Marie, abandonné son projet.

MAE Les « mots de la tribu » (Mallarmé) sont à rude épreuve dans les médias.

La représentation du dire autre ne repose alors - hormis son attribution éventuelle - que sur les opérations de reformulation (paraphrastique et/ou autonomysante). La position d'un $\mathbf{L}$ choisissant, ainsi, comme « en retrait » de catégorisation, d'ignorer les ressources lexicales offertes par cette opération, pour s'en tenir (« je ne commente pas, je ne caractérise pas les dires que je représente... ») à des

18 Comme mode de RDA à ancrage énonciatif unique (contrairement aux DD et Bivocal) et où le $\boldsymbol{a}^{\mathbf{0}}$ est l'objet dont A parle et non sa source (contrairement aux MAS, MAE), c'est-à-dire se déployant des formes en que aux formes les plus synthétiques du discours dit « narrativisé ». 
reformulations de $\boldsymbol{e}^{\mathbf{0}}$, se prête à des effets variés : neutralité, objectivité, demi-mot faisant appel à la connivence de $\mathbf{R}$...

À titre d'exemple, cette scène de préparation culinaire entre une adolescente, narratrice, et une vieille cuisinière, à la demande de qui elle vient de laborieusement découper une truffe en fines lamelles :

(37) Quand j’ai eu fini, elle a mélangé les lamelles au reste et elle a tout haché ensemble : ma truffe, le lard, le porc, le foie, le veau. Alors, ça servait à quoi toutes ces cérémonies ?

«Des cérémonies? Si tu me la haches entière, la truffe, tu me l'écrabouilles, tu me lui presse le jus, tu me la traites comme n'importe quoi et ton plat, il n'a plus d'âme ni rien du tout. » [J. Boissard, Claire et le bonheur, Livre de Poche, 1979, p. 253].

On voit que, non catégorisée, la question, au DIL-Bivocal, de la narratrice, n'est quant à son statut de pensée ou de parole, spécifiée que par le co-texte, et quant à sa tonalité - j’ironisai / je protestai / je m’agaçai / je m’amusai /je m'étonnai, etc. « confiée » au lecteur, familier des personnages.

Le procès de Julien Sorel offre une belle mise en œuvre de l'opposition entre dire catégorisé ou non : c'est sur le fond de propos (majoritairement au DI) souvent richement voire exclusivement catégorisés émanant de l'ensemble des acteurs de la « cérémonie judiciaire » - président, avocat général, avocat de Julien, témoins -, tels :

(38a) L’avocat général faisait du pathos en mauvais français sur la barbarie du crime commis [...] L'avocat encouragé adressa aux jurés des choses extrêmement fortes. [...]

Comme le président faisait son résumé [...]

que s'élève, détachée typographiquement du reste du texte, vierge de toute « préparation catégorisante »- par annonceur ou introducteur - qui pourrait en atténuer la force de rupture avec les discours qui précèdent des représentants de la société, et avec la société elle-même, la parole, formulée au DD, de Julien :

(38b) « Messieurs les jurés

L'horreur du mépris, que je croyais pouvoir braver au moment de la mort, me fait prendre la parole. Messieurs, je n'ai point l'honneur d'appartenir à votre classe [...]. » [Stendhal, Le Rouge et le noir, chap. XLI Le jugement].

Il n'en va pas de même avec le DI. Certes, le DI présente des formes non marquées, interprétatives, dépourvues, dans le cadre phrastique, de catégorisation : c'est notamment ce qu'autorise le phénomène de l'extension interprétative d'un DI marqué au-delà des bornes phrastiques ${ }^{19}$

19 Phénomène ( $c f$. chap. 9, exemples (22) à (26)) qu'il importe de distinguer des enchaînements DI-Bivocal bien connus comme : « Il dit qu'il est ravi. Quel bonheur qu'on ait pensé à lui ! ». 
(39) J'ai interviewé mon gourou pour ton problème d'arbre. Il s'est souvent occupé de petits jardins. Il dit que l'olivier de Bohème serait un bon choix ${ }_{(1)}$. Son feuillage (gris et léger) est en général heureux et contrasterait avec ta haie $\operatorname{sombre}_{(2)}$. Il a un petit développement $t_{(3)}$. Il n'est pas fragile (en tout cas pas de problème de froid dans la région) $)_{(4)}$. Par contre il déconseille le cytise, qui n'est intéressant qu'au moment de la floraison et « ingrat » le reste du temps. Voilà pour le compte rendu de mission ! À part ça ici, tout va bien [...] [corresp. privée, 05-10-2008].

Mais ces occurrences de DI interprétatif, sans catégorisation (phrases 2, 3, 4) sont, on le voit, sous la dépendance d'une catégorisation explicitée en amont (phase 1), et, de surcroît, restreinte au cas de l'assertion ${ }^{20}$, les conditions restrictives propres à ce mécanisme d'extension extra-phrastique de la catégorisation en DI étant étrangères à la liberté d'occurrence de formes sans catégorisation dans les autre modes ( $c f .(36))$.

\section{En DI, le verbe dire est déjà catégorisant}

Contrairement aux autres modes, c'est, en DI, par l'opération de catégorisation que passe la représentation de cet élément énonciatif essentiel qu'est la modalité d'énonciation. Cette « lexicalisation » obligatoire de la modalité d'énonciation en DI a pour corollaire que le DI ne connaît pas - comme le DD et le Bivocal - de « catégorisation zéro » par le verbe « neutre » dire : là où, en $\mathrm{DD}$, il dit, compatible avec des séquences « $\mathbf{e}$ » de toutes modalités

(40) Il dit : « Je viens. / Est-ce que Paul est là ? / Taisez-vous !»

en reste à ce degré zéro de la catégorisation d'un événement comme dire, il est, en DI, déjà porteur d'une catégorisation sémantique $d u$ dire comme assertion (avec que) ou injonction (avec de) - l'interrogation exigeant d'être catégorisée en « demander » :

(41) Il dit qu'il vient. ; Il dit de se taire. ; Il demande si Paul est là.

Le il dit du DD indique un fait de parole, sans analyse sémantique aucune d'un $\boldsymbol{e}^{\mathbf{0}}$ (que L peut d'ailleurs ne pas comprendre) ; le il dit que/de du DI présente, lui, obligatoirement une catégorisation sémantique du fait de parole analysé comme assertion ou injonction.

20 l demande si/ordonne de peuvent, comme l dit que être suivis de subordonnées détachées, avec reprise de l'élément subordonnant - $l$ demande si P. Si Q. Si $R$. -, en revanche, les suites $l$ demande si $P$. $Q$. R. ne peuvent pas donner lieu au rattachement interprétatif de $Q$. $R$. à la catégorisation initiale par extension, comme avec $l$ dit que P. Q. $R$. 


\section{Le DI peut reposer sur la seule catégorisation}

Là où tous les autres modes comportent, nécessairement, une image distincte de $\boldsymbol{e}^{\mathbf{0}}$, par reformulation, le DI présente des représentations de $\boldsymbol{e}^{\mathbf{0}}$ absorbées tout entières dans la catégorisation : on l'a vu en (10b), ou (25a, b, c c) ${ }^{21}$, et les textes cités ci-dessous en $\mathbf{5}$ en offrent de nombreux exemples. Dans un DI conçu comme espace de pondération variable entre les deux opérations de catégorisation et reformulation paraphrastique, apparaît un pôle de la catégorisation, correspondant à ce que l'on appelle - avec des extensions diverses - « discours narrativisé ». On notera seulement ici que sa caractérisation, comme traitant le discours " comme un événement parmi d'autres ", " un procès quelconque " (Genette, 1972 :192), passe à côté du caractère spécifiquement métalangagier de la catégorisation : l'analyse du sens d'un acte de parole $\boldsymbol{a}^{\mathbf{0}}$ que comporte un élément catégorisant n'est pas moins métalangagière - c'est-à-dire propre à un objet non pas «quelconque » mais langagier - que ne le sont les traitements par paraphrase ou autonymie... Et la RDA de pure catégorisation n'est pas synonyme non plus de cette moindre précision de la représentation que l'on prête volontiers au « discours narrativisé », comme le montrent le couple (42 a-b), l'énoncé (43) ou, ci-dessus, $(30,31)$ :

(42) a Il a dit que le film de X était très mauvais.

b Il a signé une critique assassine du film de X.

(43) Paul a longuement évoqué de façon pittoresque les péripéties de son expédition malheureuse au Népal.

\section{Discours, textes et types de catégorisation}

La place faite au sein d'un discours à l'opération de catégorisation, envisagée notamment sous l'angle de la mise en œuvre des ressources lexicales disponibles - entre minimalisme répétitif et déploiement de variété - est une variable pertinente dans la caractérisation différentielle des discours, genres et choix singuliers d'écriture. Des stéréotypes de catégorisation du dire apparaissent comme «faisant partie » de genres fortement normés, tels que procès verbaux de conseils et assemblées divers, rapports de soutenance de thèse, contes pour enfants.

21 Comme, au chap. 4, les exemples (21, 22). 
Dans le français oral « ordinaire »- corpus d'entretiens non directifs ${ }^{22}$ ou parler «spontané $~^{23}$ - l'écrasante domination du verbe dire a été régulièrement mesurée ; on a, de même, dégagé la répétitivité lexicale - très haute fréquence d'un petit nombre de lexèmes : dire, déclarer, affirmer, estimer, annoncer, expliquer, ajouter, souligner, préciser, indiquer, par ordre décroissant - comme une caractéristique de la presse d'information ${ }^{24}$.

Et, par comparaison, la dispersion lexicale dans la catégorisation des dires qu'affiche le Verbatim des années Mitterrand de J. Attali ${ }^{25}$, peut apparaître comme une « manifestation d'auteur » :

(44) Mercredi 31 août 1983 / [... immigration] Georgina Dufoix et Gaston Deferre s'opposent. Deferre met l'accent sur l'expulsion des clandestins ; le Président lui donne raison./ On discute d'un programme concernant la famille, [...] Bérégovoy et Dufoix proposent la création [...]./ [...] Tragédie : cette nuit, un Boeing 747 sud-coréen de la KAL est abattu au-dessus de la Sibérie [...]. Reagan réagit vite et très violemment. [...] Nos militaires se perdent en conjectures [Verbatim I, Livre de poche, p. 748].

Du côté des mémorialistes comme Retz ou Saint-Simon, c'est une forte densité de catégorisations variées, pittoresques, subjectives, des dires qui anime la représentation de la parole telle que les lieux de pouvoir en offrent la comédie - tel ce compte-rendu d'un conseil chez la reine en 1648, en pleine « émotion populaire », d'une « journée des barricades », réponse des Parisiens à l'arrestation par la reine du conseiller Broussel :

(45) Le maréchal de La Meilleraye, qui vit que La Rivière, Bautru et Nogent traitaient l'émotion de bagatelle, et qu'ils la tournaient même en ridicule, s'emporta : il parla avec force, il s'en rapporta à mon témoignage. Je le rendis avec liberté, et je confirmai ce qu'il avait dit et prédit du mouvement. [...] la Reine se mit en colère, en proférant, de son fausset aigri et élevé, ces propres mots : « Il y a de la révolte à s'imaginer que l'on se puisse révolter ; voilà les contes ridicules de ceux qui la veulent. [...]. » Le Cardinal, qui s’aperçut à mon visage que j'étais un peu ému de ce discours, prit la parole, et, avec un ton doux, il répondit à la Reine : «Plût

22 Mochet (1994 : 258 sq.), dans 1156 formes de RDA, relève l'emploi de dire dans 97\% des DD (majoritaires dans le corpus) et plus de 60\% des DI conjonctionnels (recourant à demander dans $23 \%$ des cas). Seuls les 34\% de RDA classées comme « narrativisées » présentent une réelle variété lexicale.

23 Vincent et al. (1997 : 82) notent que « 70\% des discours rapportés [près de 5000 occurrences de DD et DI] débutent par ce verbe [dire] ».

24 Monville-Burston (1993).

25 Dans une dizaine de pages - du 1.08.1983 au 5.09.1983 - les 82 occurrences de catégorisation passent par 54 lexèmes différents ; dire n'apparaît que deux fois ; 7 lexèmes seulement (écrire, lettre, demander, réponse, refuser, proposer, accord) figurent plus de deux fois ; 37 lexèmes ont une occurrence unique. 


\begin{abstract}
à Dieu, Madame, que tout le monde parlât avec la même sincérité que parle Monsieur le Coadjuteur ! [...]. » La Reine, qui entendait le jargon du Cardinal, se remit tout d'un coup : elle me fit des honnêtetés, et j'y répondis par un profond respect, et par une mine si niaise, que La Rivière dit à l'oreille à Bautru, de qui je le sus quatre jours après : « Voyez ce que c'est que de n'être pas jour et nuit en ce pays-ci. Le coadjuteur [...] a de l'esprit : il prend pour bon ce que la Reine lui vient de dire. » [Retz, Mémoires, $2^{\mathrm{e}}$ partie].
\end{abstract}

Apparaît aussi, comme un « type » de RDA, régulièrement associé (notamment dans le roman) à une profusion de paroles - collective, débordante, répétitive, vide, ordurière - une catégorisation riche, à dominante nominale et tendanciellement exclusive des autres opérations métalangagières, fonctionnant, résumante et lissante, comme « sauvegarde » du rythme et de l'intérêt du récit, et éventuellement de sa bienséance, comme dans ${ }^{26}$ :

(46) Toutes sortes de propos s'ensuivirent : calembours, anecdotes, vantardises, gageures, mensonges tenus pour vrais, assertions improbables, un tumulte de paroles qui bientôt s'éparpilla en conversations particulières [Flaubert, L'Éducation sentimentale, II 1].

(47) Tout ce que sa mémoire enflammée par l'alcool contenait de grossièretés, d'obscénités, d'insultes il le vomissait sur les deux bossus. Ce débordement d'outrages immondes, d'affronts sanglants, de railleries parfois cocasses déferlait contre la boutique [...] [Henri Bosco, Antonin].

(48) [...] la marquise [...] lança sur Camille un regard plein de haine [...] et trouva, sans les chercher, les flèches les plus acérées de son carquois. Camille écouta froidement et en fumant des cigarettes cette tirade furieuse qui pétilla d'injures si mordantes qu'il est impossible de les rapporter. Béatrix, irritée par le calme de son adversaire, chercha d'horribles personnalités dans l'âge auquel atteignait Mlle des Touches [Balzac, Béatrix, Folio, p. 260].

À l'inverse, le choix narratif peut être celui de la catégorisation minimale : c'est celui, affirmé, du récit auto-diégétique de L'Étranger, dans lequel l'enregistrement « déshabité », neutre, de la succession des événements - ceux « de parole », comme les autres - passe, très majoritairement, aussi loin que possible de tout mouvement interprétatif ou affectif, par des suites (DD ou DI, à source je/il alternés) de dire, demander, répondre, parler... :

(49) L'employé des pompes funèbres m’a dit alors quelque chose que je n'ai pas entendu. En même temps, il s'essuyait le crâne avec un mouchoir qu'il tenait dans sa main gauche, la main droite soulevant le bord de sa casquette. Je lui ai dit : «Comment ? " Il a répété en montrant le ciel : «Ça tape. » J'ai dit : « Oui. » Un peu après il m’a demandé : «C'est votre mère qui est là ? » J’ai encore dit : « Oui. » « Elle était vieille? » J'ai répondu « Comme ça » parce que je ne savais pas le chiffre exact. Ensuite, il s'est tu. [p 26]

26 Cf. ci-dessus chap. 4 note 15, p. 109 sur le « jarnicotonnage »; voir aussi les analyses de Hamon (2000) sur le jeu du « métalangage allusif » chez Zola, entre retranscription de « paroles crues » et catégorisations « pudiques» (p. 191-192, notamment). 
Sur le quai, pendant que nous nous séchions, elle m’a dit : « Je suis plus brune que vous.» Je lui ai demandé si elle voulait venir au cinéma, le soir. Elle a encore ri et m’a dit qu'elle avait envie de voir un film avec Fernandel. Quand nous nous sommes rhabillés, elle a eu l'air très surprise de me voir avec une cravate noire et elle m’a demandé si j'étais en deuil. Je lui ai dit que maman était morte. Comme elle voulait savoir depuis quand, j’ai répondu : « Depuis hier. » Elle a eu un petit recul, mais n'a fait aucune remarque. J'ai eu envie de lui dire que ce n'était pas ma faute, mais je me suis arrêté parce que j'ai pensé que je l'avais déjà dit à mon patron [A. Camus, L'Étranger, Livre de poche, p. 32].

Relevant d'une autre tonalité, le minimalisme de la catégorisation est plus radical encore dans Yann Andréa Steiner de Marguerite Duras (1992). Ce texte fait alterner, en contrepoint, les dialogues (autobiographiques) (a) de la narratrice avec Yann, son compagnon, au sujet du livre « arrêté » qui devait raconter l'histoire de Théodora Katz, déportée, revenue... et ceux qui adviennent (b), sur la plage où s'ébat une colonie de vacances, entre «l'enfant qui se tait », dans l'épouvante de dire le massacre des siens par les Allemands et une jeune monitrice, juive comme lui : dialogues douloureux et salvateurs, où le dire est « de vie et de mort », et dont la vérité, nue, est quasi exclusivement portée par la litanie (en DD comme en DI) des j'ai dit/vous avez dit, elle/il dit, accompagnée seulement de écrire, demander, répondre, ajouter, parler, répéter :

(50) (a) [...] vous avez dit: / -Vous n'écrirez jamais l'histoire de Théodora ? / J'ai dit que je n'étais jamais sûre de rien quant à ce que j'allais ou non écrire. / Vous n’avez pas répondu. / J'ai dit : / - Vous aimez Théodora. / Vous n'avez pas souri, vous avez dit dans un souffle : / - Théodora c'est ce que j'ignore de vous, j'étais très jeune. Tout le reste je le sais. J'attends depuis trois ans que vous écriviez son histoire. / J'ai dit : / - Je sais mal pourquoi je ne peux pas écrire l'histoire de Théodora. / J'ai ajouté : / - C'est trop difficile peut-être, c'est impossible de savoir. [p. 25]

(b) [...] et elle lui dit de bien écouter tous les bruits de la nuit. Que c'est l'été de ses six ans. Que jamais plus dans sa vie ce chiffre ne reviendra. [...]. Elle lui dit que lorsqu'il aura seize ans, à cette date d'aujourd'hui il pourra venir, qu'elle sera là à cet endroit ici de la plage mais à une heure plus tardive, vers minuit. Il dit qu'il ne comprend pas très bien ce qu'elle dit mais qu'il viendra. / Elle dit qu'elle, elle le reconnaîtra, qu'il devra l'attendre face à la rue de Londres. Qu'il ne peut pas se tromper. / Elle dit : On fera l'amour ensemble toi et moi. / Il dit oui. Il dit pas qu'il comprend pas. [p. 75] [M. Duras, Yann Andréa Steiner, Folio, le / correspond aux alinéas].

Trois occurrences - dans tout le texte - de lexèmes plus fortement catégorisants, font « accident » sur le fil continu de ces dire. L'une marque le moment où le dire de la jeune fille au jeune garçon se fait serment :

(51) «C'est à ce moment là que la jeune fille avait promis de l'emmener avec elle, de toute façon, qu'elle le jurait à lui, que jamais jamais elle le laisserait, que jamais jamais elle ne l'oublierait. » [p. 108]. 
Pour les deux autres, il est frappant de noter que ce sont des propos rejetés contextuellement du côté du faux, de l'artifice, du mensonge, qui dérogent - catégorisés en conseils (a) ou refus (b) - au dépouillement du dire :

(52) a Je vous ai dit aussi que je n’arrivais pas du tout à le lire, que Roland Barthes pour moi c'était le faux de l'écrit et que c'était de cette fausseté qu'il était mort. Je vous ai dit plus tard que Roland Barthes, un jour, chez moi, m’avait gentiment conseillé de « revenir » au genre de mes premiers romans « si simples et si charmants » comme Un Barrage contre le Pacifique, Les Petits Chevaux de Tarquinia, Le Marin de Gibraltar. J'ai ri. Vous avez dit qu'on n'en parlerait plus jamais. Et j'ai deviné que vous étiez rassasié de ce brillant auteur [p. 19].

b [les jours de pluie] Les cafés fonctionnent portes fermées. Ils refusent de servir des cafés aux familles entières. C'est trop bon marché. Ils disent que leur percolateur est en panne [...] [p. 60].

Enfin, un bel exemple de travail de la différence des types de catégorisation dans un texte est fourni par le récit du procès Champmathieu dans Les Misérables, mettant en scène la violence qui s'exerce, par le langage, dans ce lieu de « justice » : ainsi, aux catégorisations sémantico-rhétoriques dont la richesse ostentatoire est assortie à l'éloquence du barreau :

(53) L'avocat général répliqua au défenseur. Il fut violent et fleuri [...]. Il félicita [...]. [...] Ici, par une habile antonomase, remontant aux sources et aux causes de la criminalité, l'avocat général tonna contre l'immoralité de l'école romantique [...]. Ces considérations épuisées, il passa à Jean Valjean lui-même. [...]. Description de Jean Valjean. Un monstre vomi, etc. Le modèle de ces sortes de descriptions est dans le récit de Théramène [...]. La description achevée, l'avocat général reprit, dans un mouvement oratoire fait pour exciter [...] [Victor Hugo, Les Misérables, I.VII.9].

répond, représenté par les catégorisations minimales parler, paroles, dire ${ }^{27}$, le dénuement langagier d'un accusé, qui a écouté la plaidoirie « bouche ouverte, avec une sorte d'étonnement [...] qu'un homme pût parler comme cela », et dont la parole est, de part et d'autre d'une longue tirade au DD, saisie comme en deçà des catégories sémantiques (espèces d'affirmation), au plan de sa réalisation physique (rythme, timbre...) et " emportée », par un jeu serré de métaphores et comparaisons, vers le corporel non langagier (hoquets, gestes d'un bûcheron) et le phénomène naturel (éruption, s’échapper...) :

27 Cette opposition entre les deux types de catégorisation, par le $\mathbf{L}$ narrateur, du dire des deux $\boldsymbol{l}$ est redoublée par les catégorisations métalangagières internes à leurs propos (RDA ou ARD) : pour l'un accorder, prendre acte, contester, décerner, nier, opposer ; pour l'autre j'ai à dire ça ; je dis vrai, vous n'avez qu'à demander ; je vous dis monsieur Baloup ; les maîtres [...], ils disent que cela perd du temps. 
(54) [...] il se mit à parler. Ce fut comme une éruption. Il sembla, à la façon dont les paroles s'échappaient de sa bouche, incohérentes, impétueuses, heurtées, pêle-mêle, qu'elles s’y pressaient toutes à la fois pour sortir en même temps. Il dit : / J'ai à dire ça. Que j'ai été charron, même que c'était chez monsieur Baloup. [... 45 lignes].

L'homme se tut, et resta debout. Il avait dit ces choses d'une voix haute, rapide, rauque, dure et enrouée [...]. Les espèces d'affirmation qu'il semblait jeter au hasard devant lui, lui venaient comme des hoquets et il ajoutait à chacune d'elles le geste d'un bûcheron qui fend du bois [Victor Hugo, Les Misérables, I.VII.10]. 